\title{
Learning and Bayesian Updating in Long Cycle Made-to-Order (MTO) Production
}

\author{
K. Womer ${ }^{1}$, J. Camm², C. Osterman ${ }^{3}$, \\ R. Radhakrishnan ${ }^{4}, \mathrm{H} \cdot \mathrm{Li}^{1}$
}

${ }^{1}$ University of Missouri - St. Louis

${ }^{2}$ Wake Forest University

${ }^{3}$ Naval Personnel Research Study and Technology

${ }^{4}$ Integral Analytics 


\section{Learning and Bayesian Updating in Long Cycle Made-to-Order (MTO) Production}

We model production planning for made-to-order (MTO) manufacturing by choosing production rate to minimize expected discounted cost incurred up to a promised delivery date. Products that are MTO are often unique and customized. The associated learning curve slope and other production parameters cannot be precisely estimated before production starts. In this paper, a dynamic and adaptive approach to estimate the effects of learning and to optimize next period production is developed. This approach offers a closed-loop solution through stochastic dynamic programming. Monthly production data are used to update the joint probability distributions of production parameters via Bayesian methods. Our approach is illustrated using historical earnedvalue data from the Black Hawk Helicopter Program. Managerial insights are obtained and discussed.

Key words: made-to-order, project management, production planning, learning, stochastic dynamic programming, nonlinear programming, incomplete information process, Bayesian updating

\section{Introduction}

Made-to-order (MTO) is a prominent manufacturing paradigm widely found in airframe, shipbuilding, machinery and defense industries among others (Wisner and Siferd, 1995). In a typical MTO setting, a manufacturer promises to deliver the ordered products, often highly customized and low in quantity, by a certain due date. In order to make production decisions to minimize expected production cost while maintaining delivery schedules, the manufacturer must be able to realistically model and estimate the relationship between production and cost. For large projects with many activities, this process requires the manufacturer to carefully specify the interrelations among the activities and the schedule and cost risks. Summarizing these data at a 
level so that progress on the entire project can be viewed, is often done using earned value analysis (EVA). This is important at high levels within the manufacture's organization but it is often the only systematic information that is available to the customer. Here we ask, "How can we best use high-level information on the progress of an MTO project to assess cost and completion risk from earned-value data?"

We focus our efforts on long cycle projects that will produce a limited number of made-toorder items. We refer to this situation as a production program. We are particularly interested in estimating parameters that determine cost and that affect production planning. In contrast to most uses of EVA, we model two characteristics of MTO programs. First, cost tends to increase at an increasing rate with the production rate, at least beyond some threshold. Thus the attempt to speed up production to make up for schedule slippage tends to increase unit cost. Second, learning generates cost savings gained from experience with the production process, with costs typically decreasing at a decreasing rate with cumulative production. Womer (1979) shows that the optimal production rate for the manager who minimizes discounted program cost will increase throughout the life of the program. Here we are concerned with uncertainty about the values of the parameters that describe these effects and about their impact on our ability to estimate cost.

It is important to estimate parameters early to assess the production schedule in anticipation of their pronounced effects. The production of an MTO product is often a non-recurring production process. As a result, the lack of prior experience creates a difficult problem for planning the production of the new product. While cost estimating relations and experience on earlier programs may provide some guidance, production planning in this situation is uncertain at best. Waiting until there is enough data available from current production (wait-and-see) to make use of classical statistical estimation techniques may give results, but they are likely to be too late to be of practical use. This apparent dilemma eliminates the possibility of decision support from classical tools (cf. (Ebert, 1976), (Womer, Gulledge and Tarimcilar, 1986)).

In this paper, we develop a stochastic dynamic programming (SDP) model that sequentially facilitates here-and-now production assessment while accounting for an incomplete information process with unknown learning and production rate parameters. Today's production decision should be made to minimize the sum of its immediate cost in the current period plus its expected 
discounted cost in future periods through a nonlinear cost function. To efficiently obtain nearoptimal solutions, we design and implement a computationally tractable approach to approximately solve the SDP model. The procedure starts with a belief about the joint probability distribution of the random parameters, the prior distribution. As information about the realized production process becomes available in the next period, the probability distribution is updated to give the posterior estimates, which can be used for the here-and-now projection of the next period. In this way we attempt to aid the decision maker who grapples with the myriad of uncertainties involved with assessing activity early in long-cycle MTO production.

Highly visible examples of MTO production are major defense acquisition programs (MDAPs). Cost and schedule overruns of these tax-funded programs continue to be a major concern in the United States. According to the U.S. General Accounting office, for the fiscal year ending 2010, ninety-eight MDAPs ran collectively $\$ 402$ billion over budget and were on average twenty-two months behind schedule (Hofbauer, et al, 2011). In a study by Christensen, a sample of 64 MDAPs indicated that the average cost overrun was $18 \%$, with a minimum of $-3 \%$ and a maximum 109\% (Christensen, 1993). As an illustrative example, we apply our methodology to available historical earned-value data from a portion of the U.S. Army's Black Hawk Helicopter Program. Monthly production data from Cost Performance Reports on the initial lot were used for our study. These data are described in Womer and Terasawa (1984) and in Al-Abedalla and Womer (1988). Computational results show that the embedded Bayesian procedure is able to significantly reduce the variance of parameter estimates after only a few months of data. This dynamic and adaptive updating procedure offers more realistic and reliable estimates of the probability distribution of unknown parameters, and can result in higher-quality estimates of production progress, next period cost, and cost at completion for the project.

This paper is organized as follows. In Section 2, we review the related research literature. Section 3 formally describes the optimization model for the addressed problem. In Section 4, we present a stochastic dynamic programming (SDP) approach combining nonlinear programming and Bayesian updating to obtain near-optimal solutions. Section 5 provides application of our methodology to the Black Hawk Program. Section 6 gives conclusions and future research directions. 


\section{Related Literature}

The learning curve originates with Wright's (1936) study of aircraft production. It describes the regular reduction in labor hours and other cost elements as successive units of a product are produced. While these reductions may only be important for the first thousand or so items, they can be crucial for describing cost behavior for limited production runs that are often the case in the aircraft industry. Deterministic optimization for MTO production planning with learning effects has been well-studied in the literature. Womer (1979) related production rate and learning to production planning using assumed values for leaning and production. That stylized model showed how to optimize the time path of production rate to minimize production cost. According to the survey of Anzanello and Fogliatto (2011), most of the existing works assume deterministic learning/forgetting parameters, but do not account for the disturbance and randomness involved in the MTO setting. Thus, they do not address the dynamic and evolving nature of the learning curve. Majd and Pindyck (1989) optimize production decisions with a learning curve and uncertain future price, but the learning curve in their model is assumed to be deterministic. A comprehensive review of production planning under uncertainty by Mula, Poler et al. (2006) indicates that the issue of an uncertain learning effect has rarely been addressed.

Production optimization where learning parameters are random was studied by Mazzola and McCardle (1997). They model randomness assuming a known probability distribution. Their model is extended in Mazzola and McCardle (1996) to address an unknown distribution of random parameters. Both infinite and finite horizon models are developed. Analytical results are obtained using Bayesian methods with stochastic dynamic programming. For the finite horizon model, they assume a two-period case to obtain an optimal production policy. The purpose of their study is to analytically characterize the structural solution rather than to provide real-time decision support for production planning. Their models rely on general assumptions about the production function and the shape of learning curve to relate stochastic production decisions to the certainty case. Their models also apply to a short cycle production environment where the decision-maker gets to observe output and cost in one period before scheduling production for the next. This is a useful start toward our case of dealing with the real world data of long cycle MTO production. 
Lu, Petersen et al. (2009) study the stochastic nature of the learning curve in a closely related production environment known as engineering-to-order (ETO). They analyze a steam boiler design and production process, and empirically characterize heterogeneous stochastic learning curves of different partners involved in ETO. Their approach, however, is purely statistical and descriptive in nature. They do not address the production planning decision.

Some recent works develop dynamic solution approaches to incorporate a learning effect in a variety of problem settings. For instance, a dynamic programming approach is proposed by Vits, Gelders et al. (2006) and Vits, Gelders et al. (2007) to optimize process and capacity change under learning. Chen (2007) and Chen (2009) consider the effect of yield uncertainty on dynamic capacity allocation in semiconductor fabrication. Dynamic models are developed to analyze the effect of learning on make/buy (Anderson and Parker, 2002) and outsourcing decisions (Tarakci, Tang et al., 2009). These works all assume deterministic learning parameters.

The literature on earned value analysis is short on attempts to address learning in the forecasting of project costs. One exception to this is the paper by Plaza and Turetken (2009) who create a decision support system that includes learning and earned value analysis. It stops short of including stochastic components and of optimizing production decisions. A recent survey of the earned-value literature Hazir (2015) concludes that, "There is a need to develop analytic methods to forecast project performance."

Our research makes the following contribution to the literature. It integrates dynamic programming and the Bayesian updating method to provide decision-support for realistic longcycle MTO production in an uncertain environment. Different from many stochastic optimization methods which assume the probability distribution of random parameters are known, our approach dynamically and adaptively estimates the unknown learning and production rate parameters by incorporating earned-value information that arrives between decision points into the decision process. Parameter estimates obtained in this way reflect the characteristics of a unique long cycle MTO process, and thus enhance the quality of production and cost estimation.

\section{Optimization Model}

Consider a firm planning production for a new program. The contract has a delivery date of $T$ for $V$ units of products. Let $q_{t}$ be the firm's production rate and $Q_{t}$ be the firm's cumulative 
production both at period $t$. . Let $x_{t}$ be the cost of resources required in $t$. Resource is assumed to be given by the following log-linear generalization of the progress function.

$$
x_{t}=B q_{t}^{\gamma} Q_{t}^{-\gamma \delta}
$$

where $B$ is a scalar, $\delta$ and $\gamma$ are the learning and production rate parameters, respectively.

Here we assume that $x_{t}$, the cost of both material and labor resources, is subject to learning so that the production theory in Womer (1979) and Gulledge, Womer et al. (1984) apply. This model and the learning curve that it generalizes has often been used to describe the labor hours required for production. It has also been used to describe the cost of all resources required, though with a lower learning rate. This idea is hardly new, the original learning curve, Wright (1936), estimated the material cost learning rate at $95 \%$.

In the MTO environment, since each program may be unique and highly customized, parameters $B, \delta$ and $\gamma$ are unknown and must be estimated from the actual production data. Our MTO production planning problem under uncertainty is:

$$
\begin{array}{cc}
\operatorname{Min} \mathbb{E}\left[\sum_{t=1}^{T} x_{t}(1+\rho)^{-t}\right](2) & \\
\text { Subject to: } \quad x_{t}=\widetilde{B} q_{t}^{\widetilde{\gamma}} Q_{t}^{-\widetilde{\gamma} \widetilde{\delta}} & t=1,2, \ldots, T \\
Q_{t}=Q_{t-1}+q_{t} & t=1,2, \ldots, T \\
Q_{0}=0, Q_{T}=V & \\
x_{t}, q_{t}, Q_{t} \geq 0 & t=1,2, \ldots, T
\end{array}
$$

The objective function (2) minimizes the expected discounted cost of program resources. The parameter $\rho$ is the discount rate per period. In Constraint (3), the true but unknown parameters $B$, $\delta$ and $\gamma$ in (1) are replaced by their random counterparts $\tilde{B}, \tilde{\delta}$ and $\tilde{\gamma}$, respectively. Constraint (4) describes the dynamics of the system. It describes the cumulative production in period $t$ as the sum of the previous period's cumulative production and the production rate in period $t$. Constraint (5) fixes the boundary condition that $V$ units be produced in the $T$ periods of the program. Constraint (6) specifies the domain of decision variables. This stylized model is 
assumed to characterize the decision process for the production planner who optimally determines production rates and the resources needed for each time period of the program. Substituting (3) into (2), the objective function can be rewritten as:

$$
\operatorname{Min} \mathbb{E}\left[\sum_{t=1}^{T} \tilde{B} q_{t}^{\widetilde{\gamma}} Q_{t}^{-\widetilde{\delta} \widetilde{\gamma}}(1+\rho)^{-t}\right]
$$

In our problem, the production period is a month and the cumulative production experience increases during the month. This raises the question of exactly what value of $\mathrm{Q}$ should be used to characterize experience during month $\mathrm{t}$. This is similar to the "true lot midpoint" problem experienced when data are available for a lot of units. To address these issues, we replace $Q_{t}$ in (7) by $\hat{Q}_{t}$, an approximation of the average experience during the month. One way to do this is to compute the cumulative production in period $t$ as the sum of the previous period's cumulative production and half of the production in $t$, i.e. to set $\hat{Q}_{t}=Q_{t-1}+q_{t} / 2$. However, this approach does not capture the nonlinear shape of the cost curve and thus provides too much credit for experience in the current period.

To obtain a better approximation for the experience curve, we follow the integral approximation method in Camm, et al. (1987). $\hat{Q}_{t}$ is the solution to the following equation:

$$
\int_{Q_{t-1}}^{Q_{t-1}+q_{t}} Q^{-\delta} d Q=\hat{Q}_{t}^{-\delta} \cdot q_{t}
$$

Solving for $\hat{Q}_{t}$ we get:

$$
\hat{Q}_{t}=\left[\frac{\left(Q_{t-1}+q_{t}\right)^{1-\delta}-Q_{t-1}{ }^{1-\delta}}{q_{t}(1-\delta)}\right]^{-1 / \delta}
$$

Substituting (9) back into (7) and using the notation for random parameters, the objective function becomes:

$$
\operatorname{Min} \mathbb{E}\left\{\sum_{t=1}^{T} \tilde{B}\left[\frac{\left(Q_{t-1}+q_{t}+0.1\right)^{1-\widetilde{\delta}}-\left(Q_{t-1}+0.1\right)^{1-\widetilde{\delta}}}{1-\widetilde{\delta}}\right]^{\widetilde{\gamma}}(1+\rho)^{-t}\right\} \text {, }
$$

where 0.1 is a perturbation factor to handle the first period's production (Camm et al. 1987). Our optimization problem now becomes minimizing (10) subject to (4) through (6). 


\section{Closed-loop Approach with Bayesian Updating}

Solution of the above optimization problem under uncertainty involves controlling a stochastic process with unknown parameters ( (Easley and Kiefer, 1988), (Lippman and McCardle, 1991)). In this section, we develop a computationally tractable solution procedure that combines both dynamic programming and Bayesian updating to obtain an approximate solution.

\subsection{Open-loop vs. Closed-loop Approach}

One way to proceed is to solve the problem once at time zero and find optimal production quantities $q_{t}$ for all time periods $t=1,2, \ldots, T$, and therefore $Q_{t}$ and $x_{t}$, which minimize total expected discounted cost. This method is known as an open-loop approach, as the solution is obtained all at one time for the entire planning horizon (cf. (Bertsekas, 2007)) assuming the probability distribution of random parameters is known. This approach is unsatisfactory for our problem, because of the initial uncertainty about the production parameters and the inability of the approach to make use of new information as it becomes available during production. Without such information, the solution would rely only on prior belief about the random parameters.

In contrast, a closed-loop approach exploits the opportunity for sequential decisions in the problem via dynamic programming (DP) (Bertsekas, 2007). It resolves the problem each time period and obtains a policy (decision rule) that maps a stage-state pair in a DP formulation to a decision. It is a dynamic and adaptive approach, more flexible than an open-loop approach. Furthermore, the sequential nature of the closed-loop approach makes it possible to employ information arriving between decision points to enhance solution quality.

We extend the deterministic DP models of Womer et al. (1986) to formulate the addressed optimization problem as a stochastic dynamic program (SDP) (Ross, 1983). Define a stage $t \in T$ as the start of a period among the set $T$ of all time periods in the planning horizon. Let the state $S(t)$ of a stage $t$ be the current cumulative production, $Q_{t}$. The decision to be made at $t$ is the production quantity, $q_{t} \geq 0$. The system dynamics can be described by a transition function of (4), where the current state $Q_{t}$ is a function of the previous state, $Q_{t-1}$, and the current production $q_{t}$. The probability of transitioning from the current state $S(t)$ to the next state $S(t+1)$ is denoted by $p[S(t+1) \mid S(t)]$, which is governed by the joint probability distribution 
of the random parameters $\tilde{B}, \tilde{\delta}$ and $\tilde{\gamma}$. The overall objective function of the SDP is to minimize total expected discounted cost for all subsequent stages.

The closed-loop policy can be obtained by recursively solving the following stochastic version of the Bellman optimality equation (Bellman 1957):

$$
\mathcal{C}_{t}\left(S_{t}\right)=\tilde{B}\left[\frac{\left(Q_{k-1}+q_{k}+0.1\right)^{1-\widetilde{\delta}}-\left(Q_{k-1}+0.1\right)^{1-\widetilde{\delta}}}{1-\widetilde{\delta}}\right]^{\widetilde{\gamma}}+\sum_{S(t+1)} p[S(t+1) \mid S(t)] \cdot \mathcal{C}_{t+1}\left(S_{t+1}\right)
$$

where the cost-to-go function $\mathcal{C}_{t}\left(S_{t}\right)$ at stage $t$ is the immediate cost plus the total expected discounted cost of all the future stages (including the current one). The decision $q_{t}$ made at $t$ is one that minimizes $\mathcal{C}_{t}\left(S_{t}\right)$ subject to the domain $\mathfrak{D}$ defined by Constraints (4) and (5):

$$
q_{t}=\operatorname{argmin}_{q_{t} \in \mathfrak{D}\{(4),(5)\}} \mathcal{C}_{t}\left(S_{t}\right)
$$

Optimal production decisions can be obtained by solving (12) recursively. However, the exact backward recursion approach suffers the well-known curse-of-dimensionality due to the high-dimensional solution space and a large number of possible states. This makes it prohibitively challenging to solve (12) in its exact form. To circumvent these issues, we approximate the cost-to-go function.

It is also important to note that our SDP model has an incomplete information process, where the decision-maker does not know a priori the probability distributions of random parameters $\tilde{B}, \tilde{\delta}$ and $\tilde{\gamma}$. But information about their true probability distribution can be gleaned from the production data becoming available between decision points.

The design of a closed-loop algorithm must handle the following computational issues: (a) decision variables with high-dimensional solution space; (b) a large number of possible states; and (c) random parameters with unknown probability distributions. (a) and (b) make it prohibitively challenging to compute the exact form of cost-to-go function in (12). To overcome this curse-of-dimensionality, we design schemes to approximately solve (12). To handle (c), we employ Bayesian updating to learn about the distribution of random parameters during the solution process. The Bayesian method is described next, followed by an integrated procedure embedding Bayesian updating to approximately solve the SDP. 


\subsection{Bayesian Updating}

Figure 1 depicts a typical MTO production setting. Information flow is represented by dashed lines. The production planning decision $q_{t}$ and the corresponding budgeted cost of work scheduled $B C W S_{t}$ in period $t$ are obtained based on the current realized production level and estimates of learning and diminishing returns using the data available up to period $t-1$. The plan is then implemented, with exogenous disturbance arriving between periods $t$ and $t+1$. The disturbance makes the actual production level $q_{t}^{\prime}$ and the actual cost of work performed ACW $P_{t}$ deviate from the planned quantities. After the exogenous disturbance is observed, i.e. the production and cost data in period $t$ become available, one may use Bayesian methods to update the belief about the random parameters, the prior distribution. The updated, posterior distribution, is then used with the realized production level and cost to plan production in period $t+1$ and beyond.

\section{Place}

\section{Figure 1. Typical information process of an MTO production.}

\section{about here.}

The production cost $\tilde{C}_{t}$ in $t$ is a function of random parameters $\tilde{B}, \tilde{\delta}$ and $\tilde{\gamma}$, the cumulative production level $Q_{t}$ and instantaneous production level $q_{t}$ :

$$
\tilde{C}_{t}=\tilde{B}\left[\frac{\left(Q_{t-1}+q_{t}+0.1\right)^{1-\widetilde{\delta}}-\left(Q_{t-1}+0.1\right)^{1-\widetilde{\delta}}}{1-\widetilde{\delta}}\right]^{\widetilde{\gamma}}
$$

Each period, an observation on that period's production cost, $A C W P_{t}$, is used to update the model parameters. The random parameters in (13) are estimated with relationship:

$$
A C W P_{t}=\hat{C}_{t} \cdot \varepsilon_{t},
$$

where $\varepsilon_{t}$ captures the random disturbance assumed to be log-normally distributed with a mean of 1. It should be noted that a classical approach would assume that the model parameters are fixed but simply unknown. In contrast, the Bayesian approach assumes that the unknown parameters are random variables, each with its own distribution. An immediate consequence is that the output of our analysis is a probability distribution rather than a point estimate. 
Let $z_{t}=A C W P_{t} / \hat{C}_{t}$ be the observed deviation between the predicted $\hat{C}_{t}$ and the observed $A C W P_{t}$. It is this ratio rather than the difference between observed cost and $\hat{C}_{t}$ that is used because we assume a multiplicative lognormal-distributed error term in the estimating relationship. The Bayesian updating process is described by:

$$
\text { Posterior }_{t}=\operatorname{Prior}_{t}(\tilde{B}, \tilde{\delta}, \tilde{\gamma}, \sigma) * \frac{1}{\sigma \sqrt{2 \pi}} e^{-\frac{1}{2}\left[\frac{\ln \left(z_{t} t\right)}{\sigma}\right]^{2}},
$$

where $\sigma$ is the standard deviation of the error term.

\subsection{Approximation Approach with Bayesian Updating}

As mentioned in Section 4.1, to obtain a computationally tractable closed-loop solution to the SDP model, our strategy is to replace the exact cost-to-go function of (11) by an approximation. We employ a combination of closed-loop and open-loop control strategies as described in Dreyfus and Law (1977) [Chapter 5]. The decision at each stage $t$ of the procedure is made based upon the current state $Q_{t-1}$ of the system, showing the dynamic and adaptive feature of a closed-loop solution approach. Instead of solving the exact cost-to-go function (12), we solve its approximate form of (16) to minimize the total expected discounted cost $\bar{C}_{t}$ for all the remaining periods at one time, which is an open-loop approach. This combined closed-loop/open-loop approach provides a computationally tractable way to solve the high-dimensional SDP model for a real life MTO production planning problem.

In each iteration, $t$, we obtain a sequence of production levels $\bar{q}_{k}$ for $k=t, t+1, \ldots, T$ by solving the following nonlinear program:

$$
\begin{gathered}
\bar{C}_{t}=\underset{q_{k}}{\operatorname{argmin}} \sum_{\omega=1}^{|\Omega|} \sum_{k=t}^{T} B^{\omega}\left[\frac{\left(Q_{k-1}+q_{k}+0.1\right)^{1-\delta^{\omega}}-\left(Q_{k-1}+0.1\right)^{1-\delta^{\omega}}}{1-\delta^{\omega}}\right]^{\gamma^{\omega}}(1+\rho)^{-t} p\left(B^{\omega}, \delta^{\omega}, \gamma^{\omega}\right) \\
\text { Subject to: } \quad Q_{k}=Q_{k-1}+q_{k} \quad \forall k=t, t+1, \ldots, T \\
Q_{T}=V,
\end{gathered}
$$

where $\Omega$ is a set of samples of the random parameters. Each sample $\omega \in \Omega$ has a probability of $p(\cdot)$ based on the current belief about the joint probability distribution, obtained by Bayesian updating in period $t$. When the joint probability distribution is continuous, the sample set $\Omega$ is 
obtained to approximate the joint probability density function ((Miller and Rice 1983), (Luceno 1999)). The objective function (16) minimizes the expected production cost for the all of the remaining periods in the planning horizon. It is an approximated form of the exact cost-to-go function of (11).

\subsection{The Approximate Dynamic Programming Procedure with Bayesian Updating}

The procedure, as shown in Figure 2, starts with decision maker's prior belief about the joint probability distribution of the random parameters. Then in each iteration (stage) $t$, the deterministic nonlinear program (16) (18) is solved to obtain the planned production levels $q_{k}$, through the rest of the program. In particular, $q_{t}$ is the predicted production in the next period with estimated production cost, $\hat{C}_{t}$. We take these to be work scheduled and budgeted cost of work scheduled $\left(B C W S_{t}\right)$, respectively. We model work performed, $q_{t}^{\prime}$ as different from work scheduled due to random, exogenous disturbances and it is $q_{t}^{\prime}$ that leads to $A C W P_{t}$ that is observed. From this, the posterior joint probability distribution is updated through (15). The procedure then proceeds to the next iteration, and terminates when $t$ reaches the number of periods $T$ in the planning horizon. Notice that while each of the $q_{k}$ 's through the end of the program are determined, it is only $q_{t}$ and its corresponding predicted cost, $\hat{C}_{t}$ that is explicitly used at this stage. This is the primary decision support that is provided by the model. Also notice that at each stage we produce an estimate of the cost at program completion conditional on $\mathrm{T}$.

Bayesian updating plays a central role in our algorithm. It transforms a stream of observable data into continually improving estimates about random parameters that help optimize cost and production estimates. On the other hand, the closed-loop framework facilitates the incorporation of the Bayesian method in an approximate dynamic programming procedure.

\section{Place}

Figure 2. Flow chart of the approximate dynamic programming procedure with Bayesian updating.

about here. 


\section{Application: The Black Hawk Airframe Production Program}

The Black Hawk was the first new helicopter to be developed by the U.S. Army after the early 1960's. It is a twin engine helicopter designed for the transport of troops and equipment into combat, for resupply of these troops, and for the associated functions of aeromedical evacuation, repositioning of reserves, command and control, and other combat support. The first airframe contract was awarded in December 1976 and production began in February of 1977.

The Black Hawk program was characterized by cost overruns, schedule slips and quantity changes during the period February 1977 - December 1982. Development costs increased because of unanticipated technical difficulties. In particular, delays in the production schedule were attributed to contractor production start-up problems, federal funding shortfalls, and the effects of other (DOD) programs procuring Black Hawk derivative airframes from the same contractor. The original schedule called for a ten-year procurement (1977-1986), with a peak monthly production rate of 15 units/month. The procurement schedule was gradually stretched out to a 16-year program with a peak monthly rate of 8 units. Cost increases and production delays were among the factors causing procurement schedule revisions. This history is documented by Gates (1983). Some notable events impacting the program schedule and cost include:

E1. An unfavorable schedule variance during April (1977) was primarily caused by late start-up of tool fabrication for transmission and rotor system parts. Budget performance continued to be favorable in all functional categories except Engineering.

E2. Although the dollar value of the schedule variance increased during July (1977), schedule position remained approximately one month behind the internal planning schedule. The schedule variance continued to be attributable to tool fabrication and procurement. Expenditure through July represented 4.1 percent of contract target cost, while work on contract was 4.4 percent complete.

E3. The schedule position at February 28, 1978 remains approximately six weeks behind the internal production setback schedule. Costs remain within variance thresholds. 
E4. The schedule position as of June 30, 1978 is approximately ten weeks behind the internal production set-back schedule. During June, significant deliveries of late tools were experienced, further reducing the reported behind schedule condition.

E5. The schedule position remains approximately 12 weeks behind schedule (as of September 30, 1978). As stated in the last several reports, the first production aircraft will be delivered during October 1978. Sikorsky's estimate at completion has been revised with a significant increase.

E6. The schedule position for first year production continues to remain approximately twelve weeks behind schedule. The third production aircraft was delivered on January 14, 1979. The estimate at completion has been increased again.

E7. The sixth production aircraft was delivered in March (1979). The current period favorable schedule variance reflects catch-up of past due work in the detail shops and acceleration of production line activity due to improving flow of parts and reduction of shortages. The cost variance continued to follow the unfavorable trend of recent months, and has been influenced greatly by the schedule position of first year production effort.

\subsection{The Production Data}

Cost Performance Reports on the Black Hawk Contract [00 AA J01-77-C-001] form the source data for this paper. Monthly cost data of the first production lot of fifteen aircraft produced between February 1977 and July 1979 provide thirty observations. These earned value data are described by Womer and Terasawa (1984) and Al-Abedalla and Womer (1988). Figure 3 plots the budgeted cost of work scheduled $\left(B C W S_{t}\right)$, the budgeted cost of work performed $\left(B C W P_{t}\right)$ and the actual cost of work performed $\left(A C W P_{t}\right)$ from this dataset. Cumulative sums of $B C W P_{t}$, $B C W P_{t}$, and $A C W P_{t}$ are now commonly referred to as planned value, earned value and actual cost respectively.

The difference between $B C W S_{t}$ and $B C W P_{t}$ indicates the deviation of production progress from the schedule in that period: either delay or speedup. It appears that planners of the Black Hawk program had been overly-optimistic about the production progress early in the program. That is, work performed appears to be below work scheduled nearly every month until September 1978 (production had been delayed, e.g. events E1 through E5). This is not unusual in 
large-scale MTO production, where the production environment is often not stable early in the process, but continues to improve and stabilize over time. After this period, work performed apparently exceeded work scheduled in the attempt to get back on schedule (see E7).

The difference between $B C W P_{t}$ and $A C W P_{t}$ reveals another exogenous disturbance to the cost of production. Cost estimates seem to be accurate for the first 13 months of the program, as $B C W P_{t}$ and $A C W P_{t}$ roughly coincide with each other (E1 and E2). Starting from March 1978, the cost estimates are below the actual cost of work performed. This might be due to the additional cost caused by production defects, disruptions, and overtime cost to speed up the program (E6 and E7). It is also likely that workarounds accomplished early in the program due to late tooling produced production difficulties later in the program.

\section{Place}

Figure 3. BCWS, BCWP and ACWP of the Black Hawk program.

about here.

\subsection{Implementation of Our Approach}

For this data set, the actual production level cannot be directly observed. That is, we can observe effort and its deviation from planned effort but, for many months at the beginning of a program, there is work in process on many airframes but no airframes have been completed. Therefore we estimate production rate based on $\mathrm{BCWS}_{\mathrm{t}}$ and its deviation from $\mathrm{BCWP}_{\mathrm{t}}$. Due to the exogenous disturbance on production, the realized production rate may deviate from the planned rate. The estimated value $q^{\prime}$ is inferred from the relationship at (19), with the assumption that the work scheduled was optimal. For this purpose we take $q_{t}$ as that determined the previous month.

$\frac{\hat{C}\left(q^{\prime}\right)}{\hat{C}(q)}=B C W P / B C W S=\mathrm{R}$

For a sample $\omega$ of random parameters in period $t$, (19) becomes:

$\left[\frac{\left(Q_{t-1}+q_{t}^{\prime \omega}+0.1\right)^{1-\delta^{\omega}}-\left(Q_{t-1}+0.1\right)^{1-\delta^{\omega}}}{\left(Q_{t-1}+q_{t}+0.1\right)^{1-\delta^{\omega}}-\left(Q_{t-1}+0.1\right)^{1-\delta^{\omega}}}\right]^{\gamma^{\omega}}=R_{t}$ 
Letting $A^{\omega}=\left(Q_{t-1}+0.1\right)^{1-\delta^{\omega}}$ and $D^{\omega}=\left(Q_{t-1}+q_{t}+0.1\right)^{1-\delta^{\omega}}$, we solve for $q_{t}^{\prime \omega}$ :

$q_{t}^{\prime \omega}=\left[D^{\omega} \cdot R_{t}^{1 / \gamma^{\omega}}+A^{\omega}\left(1-R_{t}^{1 / \gamma^{\omega}}\right)\right]^{1 /\left(1-\delta^{\omega}\right)}-Q_{t-1}-0.1$

These calculations correspond to similar calculations by Plaza and Turetken (2009) for their learning curve. Given this, the estimated production level over the set $\Omega$ of all samples can be computed as:

$q_{t}^{\prime}=\sum_{\omega=1}^{|\Omega|} p\left(B^{\omega}, \delta^{\omega}, \gamma^{\omega}, \delta^{\omega}\right) \cdot q_{t}^{\prime \omega}$

To illustrate this approach we assume a uniform prior distribution for the unknown parameters, and fix the standard deviation $\sigma$ in (15) to be two in the base case. The uniform priors are chosen over conjugate priors since they are straightforward for practitioners to specify and since numerical methods are needed for optimization. We compare our solution with one obtained by a rolling horizon approach without Bayesian updating.

\subsection{Computational Results for the Base Case}

We begin with the evolution of parameters as new data becomes available. In Figure 4, we illustrate the posterior pdf of the unknown parameters due to Bayesian updating at various points in the production program using equation (15). The joint posterior pdf's of the learning parameter, $\delta$, and diminishing returns, $\gamma$, are conditional on $B=11,000$. Starting from a uniform (un-informed) prior, Bayesian updating concentrates more of the probability mass for $\gamma$ at medium values but at lower values for $\delta$ after only five months (Figure 4a). This suggests a bit less effective learning but similar diminishing returns relative to the uninformed prior. That is, those early observations on cost being lower than planned production rate showed a tendency for cost to rise with production rate but not to fall as much with experience. The joint posterior pdf starts to shift in the direction of smaller $\gamma$ and even smaller $\delta$ by August of 1978 (Figure 4b). This shift continues in Figure 4c. This keeps the projected program cost stable until January, 1979.

What happened from February to April 1979 is of particular interest. Recall from Figure 3 that during this period work performed significantly exceeded the work scheduled. At the same time, actual cost of work performed was much higher than budgeted. These new data caused the joint pdfs to shift even more dramatically toward both smaller $\delta$ and $\gamma$, reflecting the change of 
the underlying structure for cost estimation. These trends accelerate afterwards until the pdf converges at the end of the lot in July 1979 (Figure 4d) giving much more precise and much lower estimates of $\gamma$ and $\delta$.

\section{Place}

Figure 4. Evolution of posterior joint pdfs of $\gamma$ and $\delta$ obtained by Bayesian updating. about here.

Figure 5. compares the optimized production decision recommendations obtained by our dynamic/adaptive solution (with Bayesian updating) and a dynamic but non-adaptive rolling horizon solution (without Bayesian updating) against the estimated production level at some of the key decision points during lot one. Both the Bayesian and the rolling horizon prescriptions show that production rates should be somewhat above the realized rates prior to September 1978. This corresponds to the notes from the Cost Performance Report (CPR) that show steady schedule slip for the program during this time period. By September 1978 the program was 12 weeks behind schedule. At this stage there seems to be a concerted effort by the program to increase production rate and our estimated rate increases dramatically, well above the optimal time path and, as noted above, at higher than forecast cost.

\section{Place}

\section{Figure 5. Production rates recommended at key dates.}

\section{about here.}

Figure 6 shows how our estimates of the total lot cost are dynamically updated during the program. The official program cost estimates, without using our methodology, are shown in the same graph. They give no indication of cost uncertainty or of real time change to the unfolding program data. The official estimate seems only to be periodically adjusted for inflation at particular points. In contrast, the evolution of program cost provided by our methodology is insightful. The initial cost estimate at completion is slightly lower than the official estimate due to the initial ranges of the parameter estimates. The Bayesian cost estimate at completion decreases during the first five months in response to the very favorable cost progress of the program at that time. It then steadily increases until September 1978. The dramatic changes in production rate at that point halts that trend for several months before the estimated cost at 
completion converges to the final actual lot cost. It is important to notice that the forecasted cost is not a strictly increasing curve. It is based on the cost that would obtain if production rate were dynamically and adaptively optimized given the information becoming available at that time. It is also sensitive to the observed cost by updating the parameter distributions. Thus early in the program, the very favorable relationship between BCWP and ACWP cause this forecast to be even lower than the original projection, but beginning in July 1977, the forecast increases and eventually passes the official forecast even after the official forecast is adjusted for inflation. The program cost forecast without Bayesian updating appears to be low and rather stable. This is because it relies on decision maker's prior belief about the uncertain parameters of learning and production which are not adjusted based on new information. We also note that the BCWP seems to never be adjusted even though the contractors estimated cost at completion is.

\section{Place}

\section{Figure 6. Dynamic and adaptive control of lot cost}

\section{about here.}

All of these estimates of completed work are based on estimates from the updated model and on the assumption that the final delivery date for the lot is fixed.

Additional insights can be gained by examining the variability of total lot cost forecasts. Figure 7 shows the $90 \%$ confidence interval of predicted total lot cost obtained by the rolling horizon approach (Figure 7a) and the adaptive approach with Bayesian updating (Figure 7b). The official lot cost estimates are also shown for comparison. The width of the non-adaptive confidence interval estimate is wide and nearly constant because it is based on the decision maker's prior belief about the random parameters without being updated. It starts showing convergence with smaller variance from October 1978. Such smaller variance, however, results from significantly less remaining production and cost due to the sudden drastic increase in the actual work performed from October 1978 (Figure 4). The adaptive confidence interval, on the other hand, gradually narrows and converges at a faster speed. It suggests that more accurate program cost estimates have been achieved through Bayesian updating during the production process. We believe that this illustrates the increase in confidence that is possible as production experience increases even in a program that was subject to substantial disruption. In both cases 
the eventual lot cost falls within the confidence interval that is estimated for most, but not all, of the time periods. This suggests that our initial estimate of $\sigma$, a measure of cost uncertainty, may be pessimistic and gives rise to the sensitivity analysis explored in Section 5.4.

Dynamic and adaptive control of the production process provides the production manager more meaningful and reliable forecasts of program cost at completion than the usual earned value analysis. Three methods are often used to estimate cost at completion using earned value analysis. The optimistic method merely continues the initial cost at completion estimate perhaps modified for revised inflation estimates. This is the sequence of estimates shown as the official program cost estimates in Figure 6 . The so called realistic estimate is found by calculating the cost performance index (CPI) as the ratio of earned value, EV (cumulative BCWP) to cumulative ACWP. The estimate is then found as

$\mathrm{EC}_{\mathrm{R}}=\left(\mathrm{EC}_{\mathrm{O}}-\mathrm{EV}\right) / \mathrm{CPI}+\mathrm{EV}$

where $\mathrm{EC}_{\mathrm{O}}$ is the current official program cost estimate. The pessimistic estimate is found using the schedule performance index SPI as the ratio of EV to cumulative BCWS. The estimate is than found as

$\mathrm{EC}_{\mathrm{P}}=\left(\mathrm{EC}_{\mathrm{O}}-\mathrm{EV}\right) /(\mathrm{CPI} * \mathrm{SPI})+\mathrm{EV}$

While the optimistic estimate tends to be stable over time, the other two estimates can fluctuate substantially. For example, in June 1977 the CPI was 1.18 while the SPI was 0.81. This gave estimates of $\$ 69 \mathrm{M}, \$ 58.8 \mathrm{M}$, and $\$ 72 \mathrm{M}$ for $\mathrm{EC}_{\mathrm{O}}, \mathrm{EC}_{\mathrm{R}}$ and $\mathrm{EC}_{\mathrm{P}}$ respectively. Note that the optimistic estimate is higher than the realistic estimate. One month later, after a low observation of ACWP, the same procedure yields estimates of $\$ 69 \mathrm{M}, \$ 70.8 \mathrm{M}$, and $\$ 95.6 \mathrm{M}$ respectively, higher for both $\mathrm{EC}_{\mathrm{R}}$ and $\mathrm{EC}_{\mathrm{P}}$ despite the favorable ACWP observation. Of course none of these estimates is made with any kind of an error bound or confidence interval. For the same months, our estimates are $\$ 47.1 \mathrm{M}$ and $\$ 46.6 \mathrm{M}$. While these estimates are low because of the very favorable performance of ACWP during prior months, the $90 \%$ confidence interval makes it clear that there is substantial uncertainty about the estimates at this early stage in the program. 
Place

\section{Figure 7.90\% Prediction intervals on lot cost.}

\section{about here.}

In addition to forecasting cost at completion, we examine monthly costs throughout the lot. For each month, $t$, we obtain a sequence of production levels $\bar{q}_{k}$ for $k=t, t+1, \ldots, T$ by solving the nonlinear program at equation (16) where $\mathrm{q}_{\mathrm{t}}$ is the solution for next month. Using $\mathrm{q}_{\mathrm{t}}$ and equation (13), $\tilde{C}_{t}$ is the predicted cost of the work to be accomplished in the next month. The distribution of $\tilde{C}_{t}$ is found using the current joint posterior distribution of the parameters and that of the error term which is assumed to be independently distributed as the lognormal with $\mu=1$ and $\sigma=2$. These values are used to determine the predictions and the five percent and ninety-five percent lower and upper bounds in Figure 8.

\section{Place}

\section{Figure 8. Prediction of monthly cost with $90 \%$ confidence intervals.}

\section{about here.}

It would be a mistake to think of Figure 8 as a control chart. With a control chart there is substantial history on a process and the current data do not influence the control limits. In our case, the Bayesian analysis uses current data to update the model and, early in the lot, this updating can be dramatic. That said, observing the ACWP compared to the predicted cost and the $90 \%$ prediction limits does provide some very useful information. In Figure 8, ACWP starts well below predicted cost for the first several months and through July 1978 it rises steadily from the lower limit through the midpoint of the prediction nearly to the upper limit. This is a classic indicator of a process that is out of control and it corroborates the written evidence that the Black Hawk was well behind schedule early in the program and that cost was increasing in the attempt to catch up. If we had a very well-informed prior distribution and if we did not update it with current information as with a control chart, then this kind of behavior would be a very strong reason for concern. As it is, this evidence should be taken as an indicator that the program has some substantial ground to be made up and, depending on exactly why there were delays, it could be a cause for concern about future costs. 


\subsection{Sensitivity Analysis}

In Figure 9, we show the sensitivity of the projected production rate to the annual discount rate (twelve times the monthly rate). As the discount rate increases the recommended production rate decreases early in the lot and shows a corresponding increase late in the lot. That is, higher discount rates induce the manager to delay production and incur costs later in the program where they will be discounted more heavily. Because the total production quantity is the same, production planned with a higher discount rate must exceed that planned with a lower discount rate late in the lot.

\section{Place}

Figure 9. Sensitivity of monthly cost to the discount rate. about here.

All of the previous results were based on the standard deviation of Black Hawk program cost, $\sigma$, equal to 2. One standard deviation in costs is equal to about $100 \%$ of the cost estimate. Here we use previous studies of airframe cost to perform a sensitivity analysis on the Bayesian analysis. The C141 program was estimated using data on labor hours expended on airframe production as reported in Womer and Gulledge (1982). In this case the model included an additive error term that was characterized by heteroscedascity. One standard deviation for this model amounted to between 7 and 63 percent of the cost estimate. Likewise the man hours for F102 program were estimated with a similar model, also with an additive error term and heteroscedascity (Womer and Gulledge, 1982). In this case the range was wider ranging from 120 to 360 percent of the cost estimate. Therefore, we conduct sensitivity analysis on $\sigma$ in the range of 100 to 300 percent.

Figure 10 shows that as $\sigma$ increases from 1.0 to 3.0, the variation of the posterior joint pdf of $\gamma$ and $\delta$ in month 30 also increases. When $\sigma$ approachs 3.0, the center of the pdf shifts to a higher value of $\gamma$, suggesting diminishing returns and a bit more learning.

\section{Place}

Figure 10. Sensitivity of $\delta$ and $\gamma$ to the standard deviation of the error term. 
about here.

We examine the marginal posterior pdfs of scalar $B$ in Figure 11. For all three values of $\sigma$ the left-shift of the pdf during the first five months forces down the estimated program cost but the shift is much less pronounced as $\sigma$ increases. This explains why the forecasted program cost decreases initially in Figures 6 and 7. The center of the pdf's then shifts to the right up to August 1978, which drives the program cost estimates up. The posterior of $B$ shows for all three values of $\sigma$ suggest the inherent variability of program cost during this first lot.

Place

Figure 11. Sensitivity of $B$ to the standard deviation of the error term.

about here.

The larger dispersion of the posterior pdfs of random parameters will result in less accuracy in total lot cost projection. Figure 12 is a comparison of the optimized program cost projection with a small $\sigma$ of 1.0 (a) and large $\sigma$ of 3.0 (b). Significantly more accurate program cost projection is asserted with $\sigma$ being 1.0, while the projection with $\sigma$ of 3.0 shows a much wider and more realistic confidence interval.

\section{Place}

Figure 12. Sensitivity of cost at completion to the standard deviation of the error term.

\section{about here.}

\section{Conclusion and Future Research}

Optimizing production rate in the MTO setting is challenged by the uncertainty of production parameters such as learning and diminishing returns. While the deterministic approach assuming full knowledge of the parameters has been extensively studied, it fails to adapt the schedule with the underlying dynamic and evolving production process. 
In this paper, we have developed a methodology to dynamically and adaptively optimize the production schedule in conjunction with estimating the production parameters. Our approach models the addressed MTO production planning problem under uncertainty as a stochastic dynamic program (SDP). A computationally tractable solution method is designed and implemented to approximately solve the high-dimensional SDP. Our method is a combination of nonlinear programming and Bayesian updating such that: (1) the joint probability distribution of production parameters is updated after the actual production cost and exogenous disturbance are observed in each iteration; and (2) each a sub-problem of optimizing the schedule of future periods is solved by nonlinear programming.

Our solution is dynamic in nature since the prescribed production rate obtained at each decision point minimizes not only the immediate cost but also expected discounted costs of all future periods. It is also adaptive in the sense that decisions are made based on the current state of the production system and the posterior joint pdf of production parameters. The use of Bayesian methods makes it possible to estimate key production parameters regarding learning rate and returns to scale early in the production process. An additional advantage of Bayesian updating is its ability to make appropriate use of the knowledge and experience of managers and subject matter experts. Managers' subjective knowledge can be employed by developing a reasonable prior probability distribution for the random parameters.

The U.S. Army's Black Hawk helicopter production data is used to examine the performance of our methodology. Computational results show that our Bayesian updating procedure is able to quickly identify the joint pdf of the production parameters unique to an MTO production process. Comparing with production schedule using the prior pdf alone, our closed-loop solution is able to recommend a production schedule best fit for the underlying production process. In the data we tested, the optimized schedule suggests higher production rate later in the production horizon, due to learning efficiency. Our solution holds promise for an effective dynamic program cost control tool for project/program managers. As more production information/data becomes available, collected and utilized by our combined optimization-Bayesian procedure, an optimized production schedule can be obtained to offer more accurate program cost projections. Our sensitivity analysis reveals that better-quality program cost forecasts can be achieved by reducing exogenous disturbance of the production process. 
Why would a program office want to use an approach like this instead of just following the usual earned value reports? First, this approach is all about risk and uncertainty. The whole premise is based on random variation of the parameters of the cost model, in addition to the randomness of the unexplained error term. This is in dramatic contrast to the fixed cost estimates $\mathrm{EC}_{\mathrm{O}}$ for the program and the estimates $\mathrm{EC}_{\mathrm{R}}$ and $\mathrm{EC}_{\mathrm{P}}$ based on $\mathrm{BCWS}$ and $\mathrm{BCWP}$. The focus on randomness leads naturally to the use of devices like confidence intervals and control charts to compare estimates with observations of cost to determine if our plans conform with cost observations. For example, early in the Black Hawk program production fell behind schedule. While BCWP was not greatly different from ACWP, the schedule slip leaves unanswered what would have been the cost of the work that was scheduled but not performed. Our stochastic model makes a prediction of the work to be performed, its predicted cost and the range of that cost. These predictions are updated with each new monthly observation and the chart in Figure 8. makes clear the work and cost predicted to be necessary to achieve lot completion goals.

The following issues could be fertile ground for future research. From the modeling perspective, our current model considers a single lot production setting. It will be interesting to extend it to allow for more than a single lot of production, where interaction between lots and the timing of production of these lots may affect the allocation of resources. Further insights might come from a more detailed model that considers crews of workers at each operating station of a production process with unique learning at each station. From the algorithmic perspective, other approximation architectures based on reinforcement learning and neural networks can be developed to offer alternative closed-loop solutions to the problem. Last but not least, the application of our combined optimization-Bayesian paradigm for decision-support under uncertainty in other application domains such as R\&D project management and planning, is of interest.

\section{Acknowledgements}

We gratefully acknowledge support from the Young Investigator Program of the Army Research Office and from the Office of Naval Research ONR grant N00014-00-1-0280 and the helpful comments of two anonymous referees. 


\section{References}

Al-Abadalla, B. K. and N. K. Womer (1988). "Production Disruption in the Military Aircraft Industry: A Study of the Black Hawk Helicopter Program," Proceedings of the First Joint Cost Analysis and Estimating Conference,

Anderson, E. G. and G. G. Parker (2002). The effect of learning on the make/buy decision. Production and Operations Management 11(3): 313-339.

Anzanello, M. J. and F. S. Fogliatto (2011). Learning curve models and applications: Literature review and research directions. International Journal of Industrial Ergonomics 41(5): 573-583.

Bellman, R. (1957). Dynamic Programming. Princeton, Princeton University Press.

Bertsekas, D. (2007). Dynamic Programming and Optimal Control. Athena Scientific.

Camm, J., J. R. Evans and K. Womer (1987). The unit learning curve approximation of total cost. Computers \& Industrial Engineering 12(3): 205-213.

Chen, T. (2007). Evaluating the mid-term competitiveness of a product in a semiconductor fabrication factory with a systematic procedure. Computers \& Industrial Engineering 53(3): 499-513.

Chen, T. (2009). Enhancing the yield competitiveness of a semiconductor fabrication factory with dynamic capacity re-allocation. Computers \& Industrial Engineering 57(3): 931-936.

Christensen, D. S. (1993), "An Analysis of Cost Overruns on Defense Acquisition Contracts." Project Management Journal 3:43-48 (September).

Dreyfus, S. E. and A. M. Law (1977). The Art and Theory of Dynamic Programming, Academic Press Inc.

Easley, D. and N. M. Kiefer (1988). Controlling a stochastic process with unknown parameters. Econometrica 56(5): 1045-1064.

Ebert, R. J. (1976). Aggregate Planning with Learning Curve Productivity. Management Science 23(2): $171-182$.

Gates, W. (1983). "UH-60 A Black Hawk: Development and Procurement Cost History," Jet Propulsion Laboratory.

Gulledge, T. R., K. Womer and J. R. Dorroh (1984). Learning and costs in airframe production: a multiple output production function approach. Naval Research Logistic Quarterly 31(1): 67-85.

Hazir, O. (2015). A review of analytical models, approaches and decision support tools in project monitoring and control. International Journal of Project Management, 33:808815.Lippman, S. A. and K. F. McCardle (1991). Uncertain search: a model of search among technologies of uncertain values. Management Science 37(11): 1474-1490.

Hofbauer, J., Sanders, G., Ellman, J. and D. Morrow, (2011) "Cost and Time Overruns for Major Defense Acquisition Programs," Defense-Industrial Initiatives Group, Center for Strategic and International Studies.

Lu, R. F., T. D. Petersen and R. L. Storch (2009). Asynchronous stochastic learning curve effects in engineering-to-order customisation processes. International Journal of Production Research 47(5): 1309-1329.

Luceno, A. (1999). Discrete approximations to continuous univariate distributions - an alternative to simulation. Journal of Royal Statistical Society: Series B 61(2): 345-352. 
Majd, S. and R. S. Pindyck (1989). The learning curve and optimal production under uncertainty. The Rand Journal of Economics 20(3): 331-343.

Mazzola, J. B. and K. F. McCardle (1996). A Bayesian approach to managing learning-curve uncertainty. Management Science 42(5): 680-692.

Mazzola, J. B. and K. F. McCardle (1997). The stochastic learning curve: optimal production in the presence of learning-curve uncertainty. Operations Research 45(3): 440-450.

Miller, A. C. and T. R. Rice (1983). Discrete approximations of probability distributions. Management Science 29(3): 352-362.

Mula, J., R. Poler, J. P. Garcia-Sabater and F. C. Lario (2006). Models for production planning under uncertainty: A review. International Journal of Production Economics 103(1): 271-285.

Plaza, M. and O. Turetken (2009). A model-based DSS for integrating the impact of learning in project control. Decision Support Systems 47:488-499.

Ross, S. M. (1983). Introduction to Stochastic Dynamic Programming. New York, Academic Press.

Tarakci, H., K. Tang and Teyarachakul (2009). Learning effect on maintenance outsourcing. European Journal of Operational Research 192(1): 138-150.

Vits, J., L. Gelders and L. Pintelon (2006). Production process changes: A dynamic programming approach to manage effective capacity and experience. International Journal of Production Economics 104(2): 473-481.

Vits, J., L. Gelders and L. Pintelon (2007). Manufacturing process changes: myopic and longterm planning. International Journal of Production Research 45(1): 1-27.

Wisner, J. D. and S. P. Siferd (1995). A survey of U.S. manufacturing practices in make-to-order machine shops. Production and Inventory Management Journal 36(1): 1-6.

Womer, K. (1979). Learning curves, production rate, and program costs. Management Science 25: 312-319.

Womer, K. (1984). Estimating learning curves from aggregate monthly data. Management Science 30(8): 982-992.

Womer, K. and T.R. Gulledge (1982). Cost functions for airframe production programs. Technical Report, Research Contract F33615-81-K-5116, Air Force Business Research Management Center, Clemson University.

Womer, K., T. R. Gulledge and M. M. Tarimcilar (1986). A comparison of several dynamic programming models of the made-to-order production situation. OR Letters 5(2): 87-92.

Womer, K and K. L. Terasawa (1984). "Program Cost Estimation with Uncertain Production Requirements." Unpublished Research Report for Arroyo Center, Jet Propulsion Laboratory. 


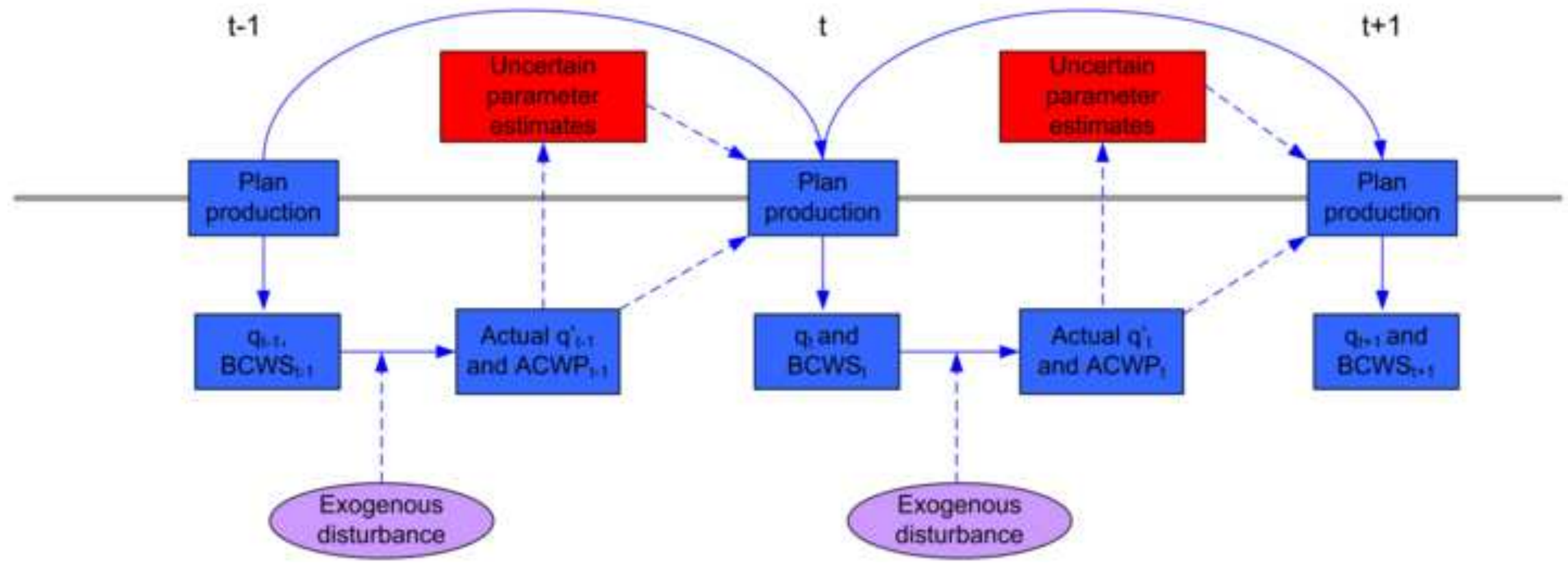




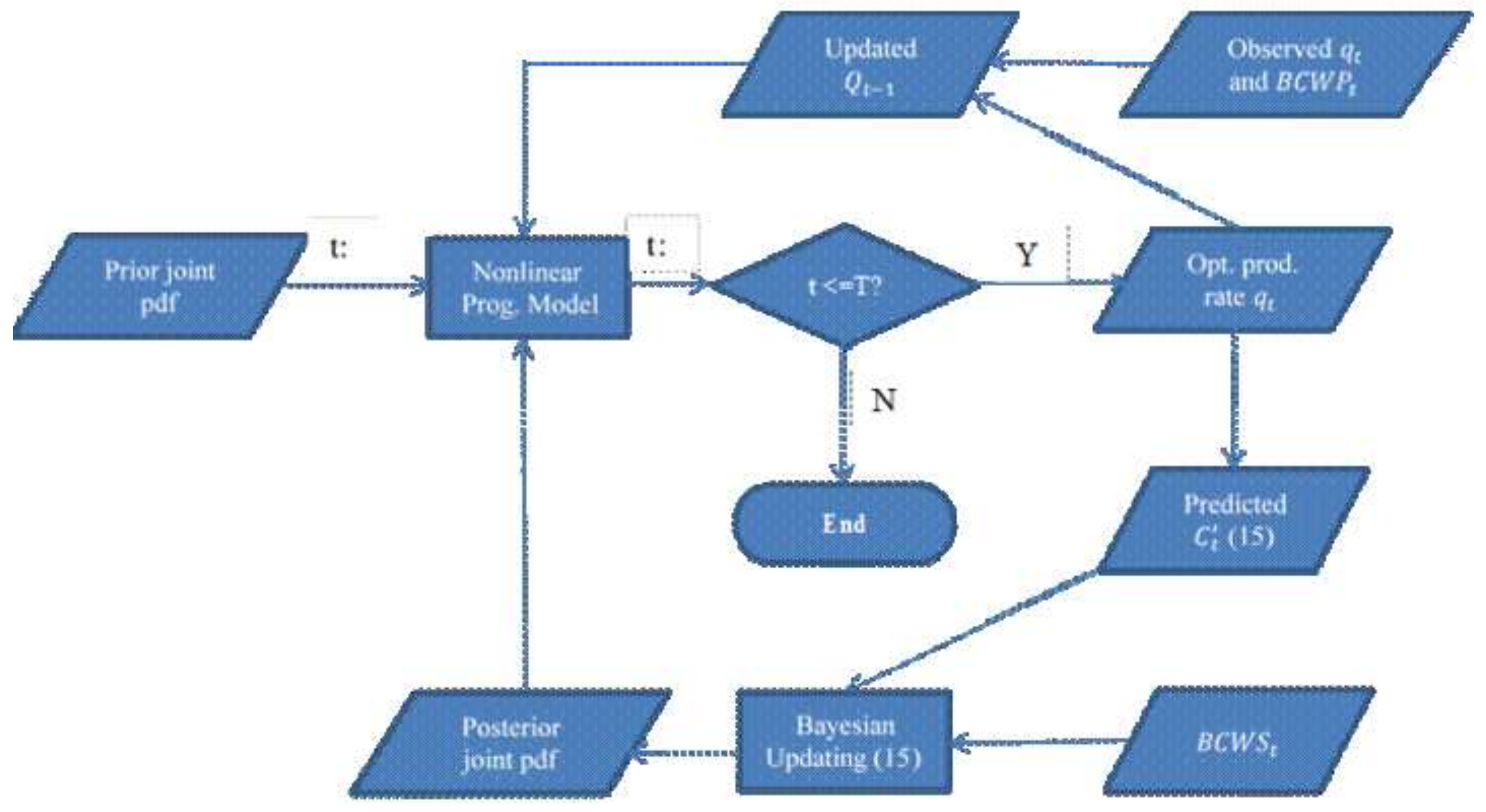



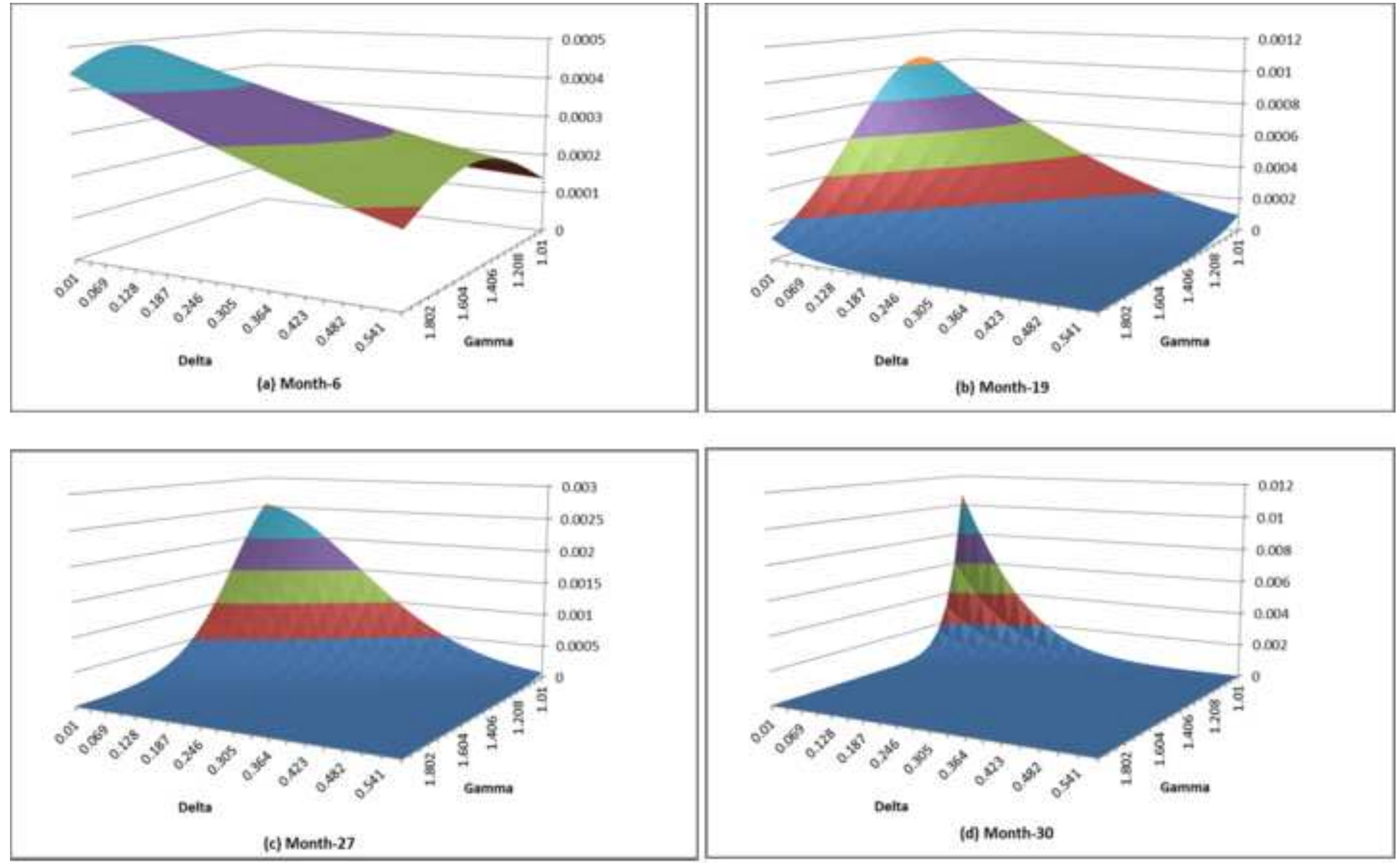


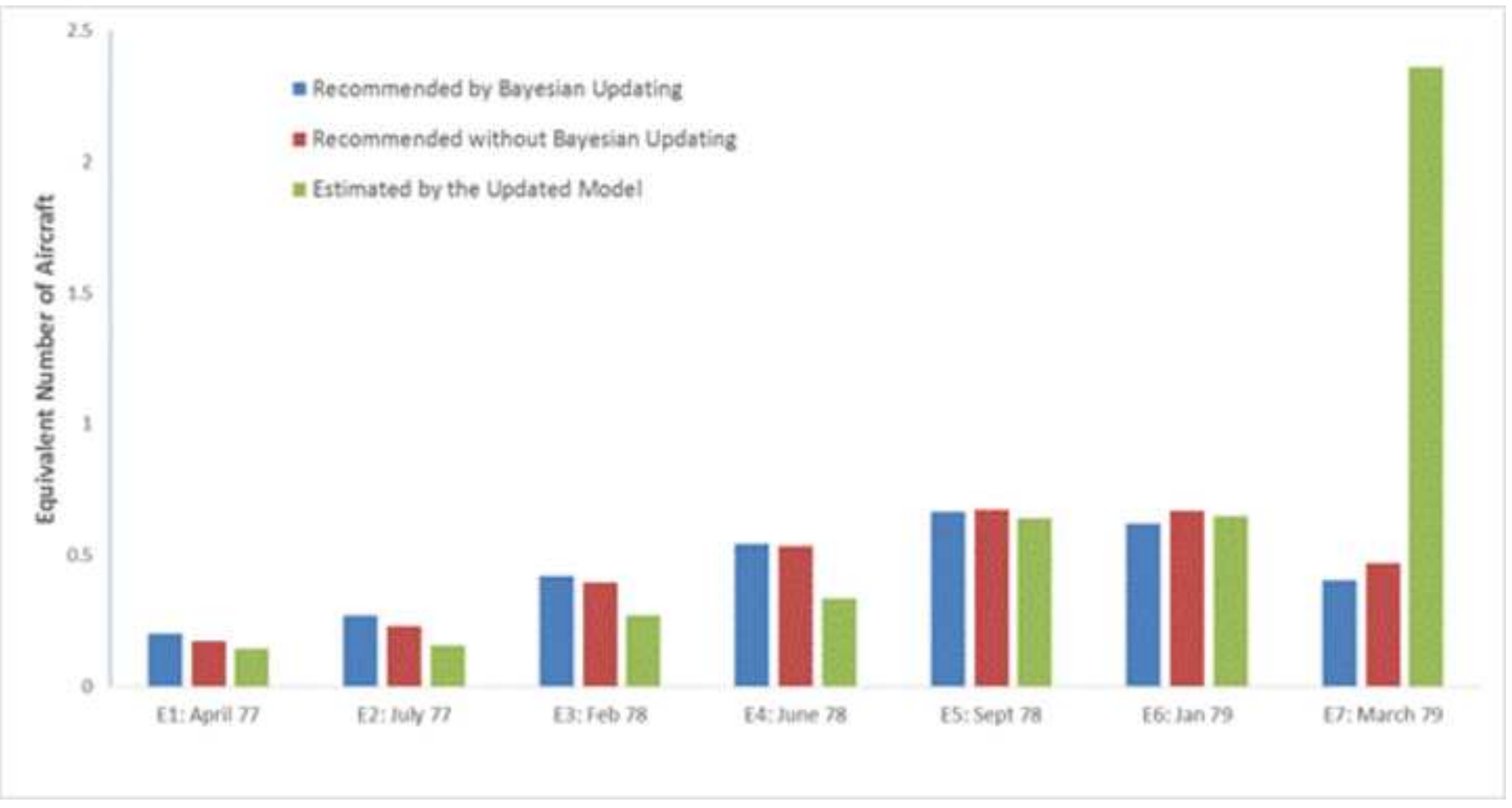

Figure(5) 


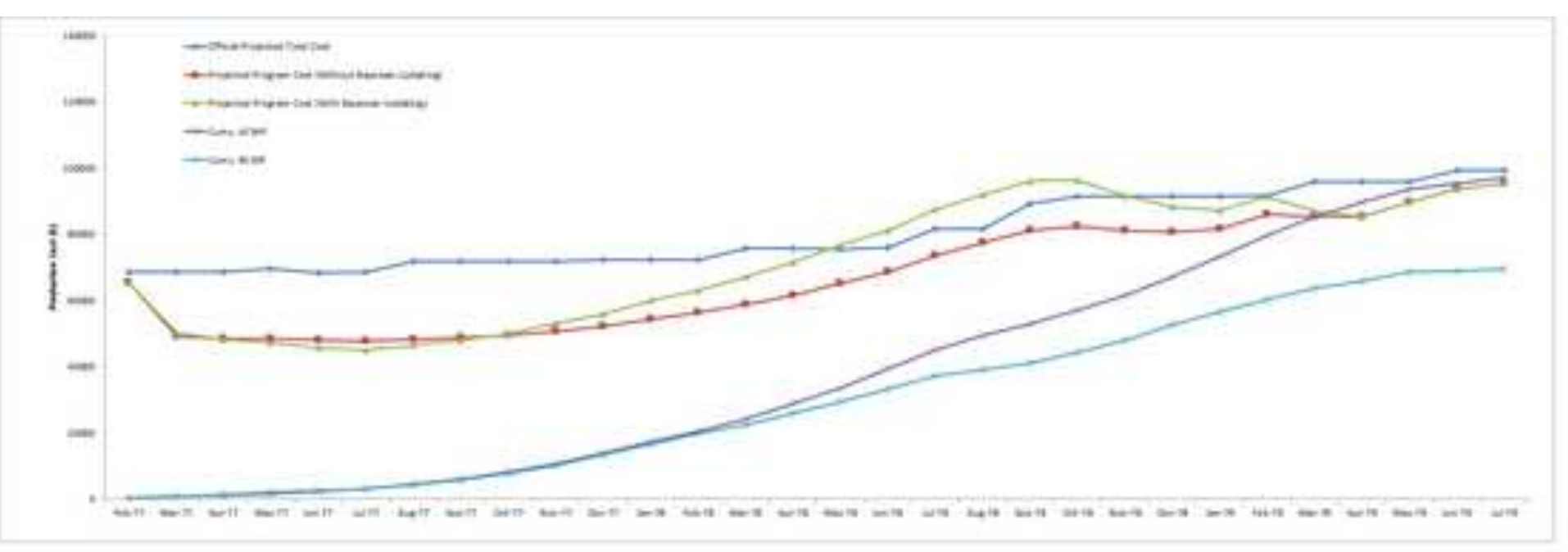




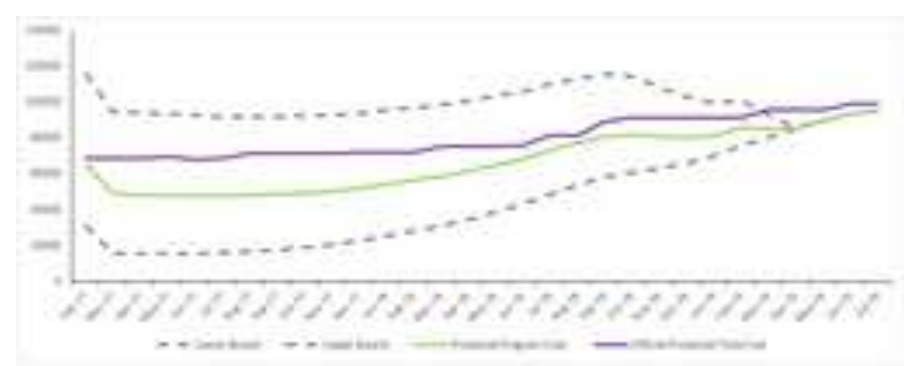

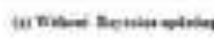

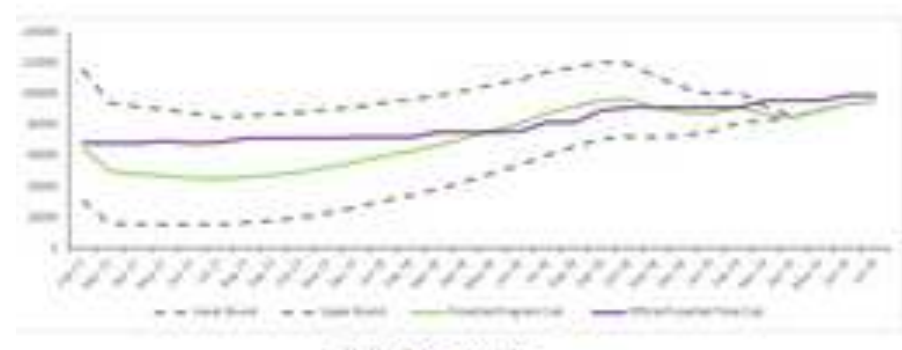

Mina biven 


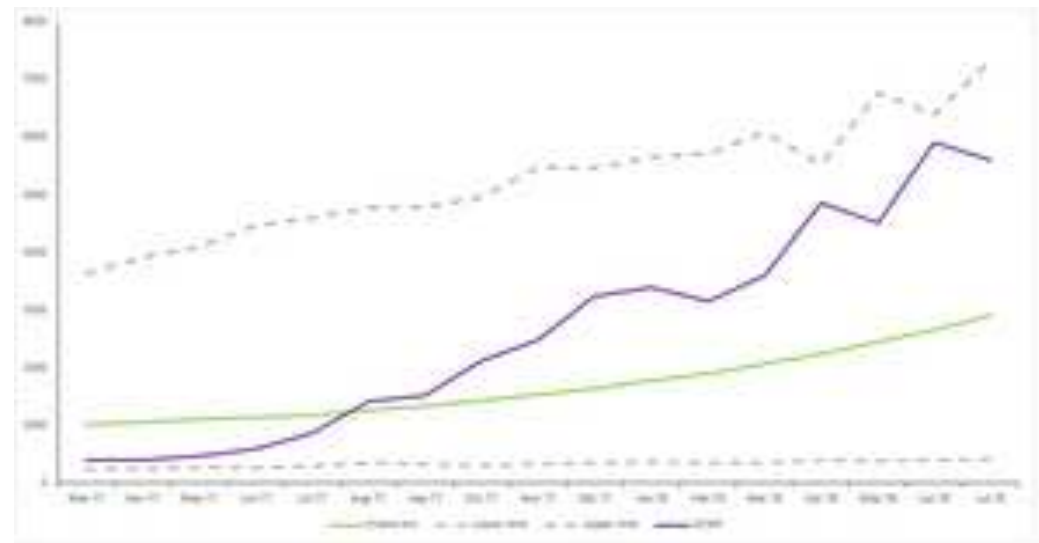




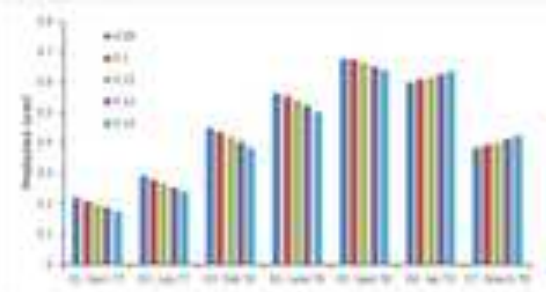

Figure

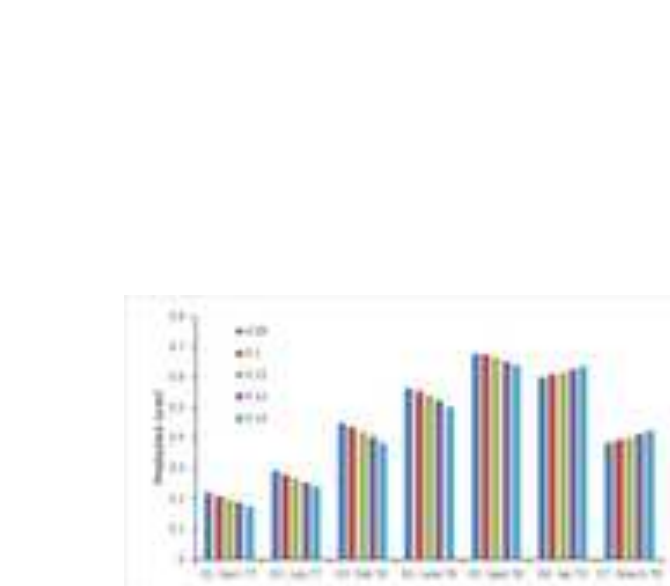

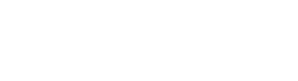

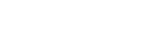
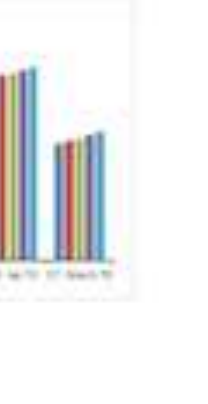

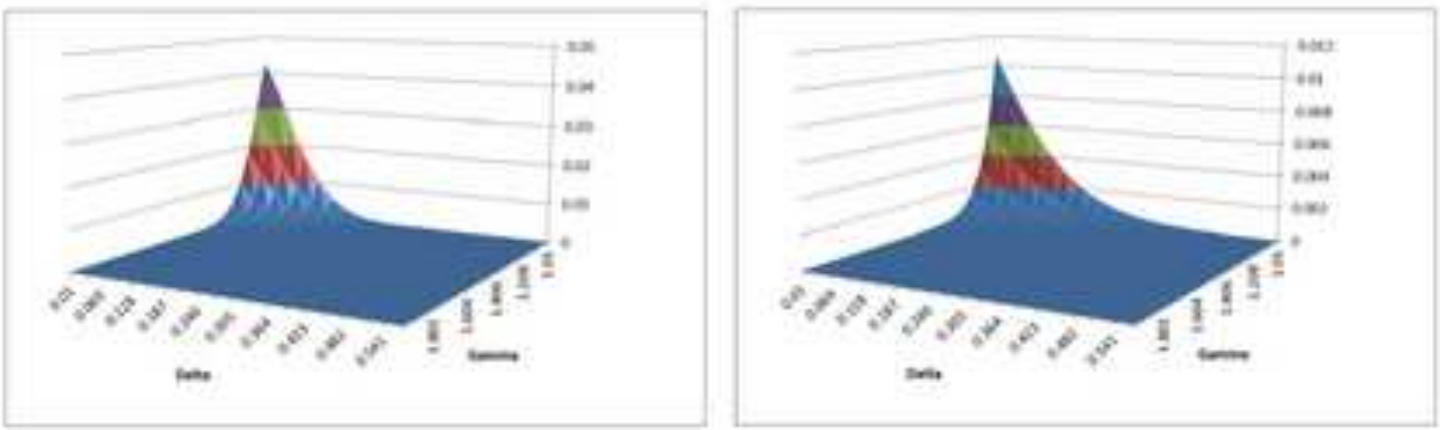

(a) Sigma $=1.0$

(b) Sigma $=2.0$

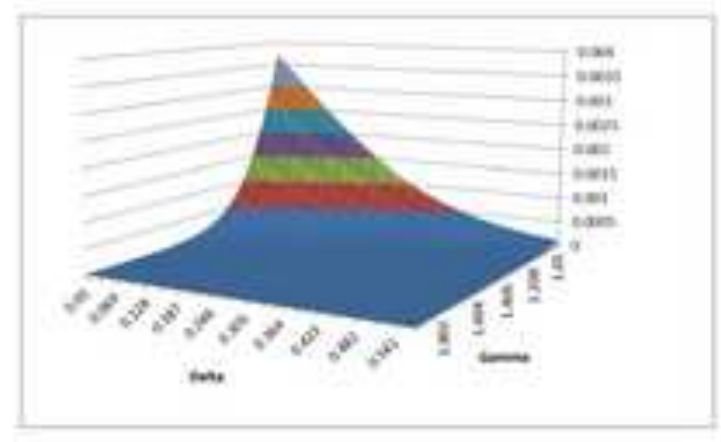

(c) Sigma $=3.0$ 

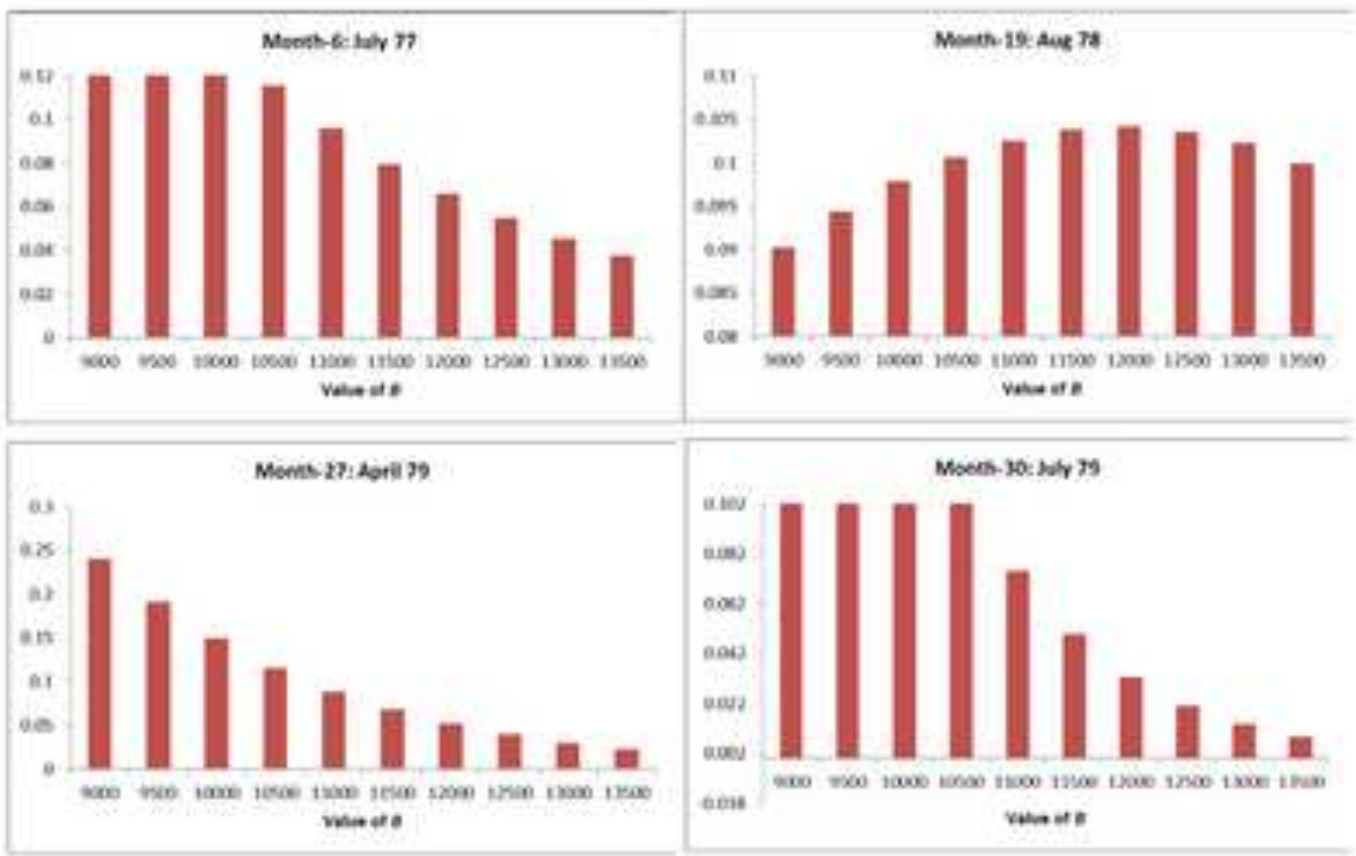

(a) Sigma $=1.0$
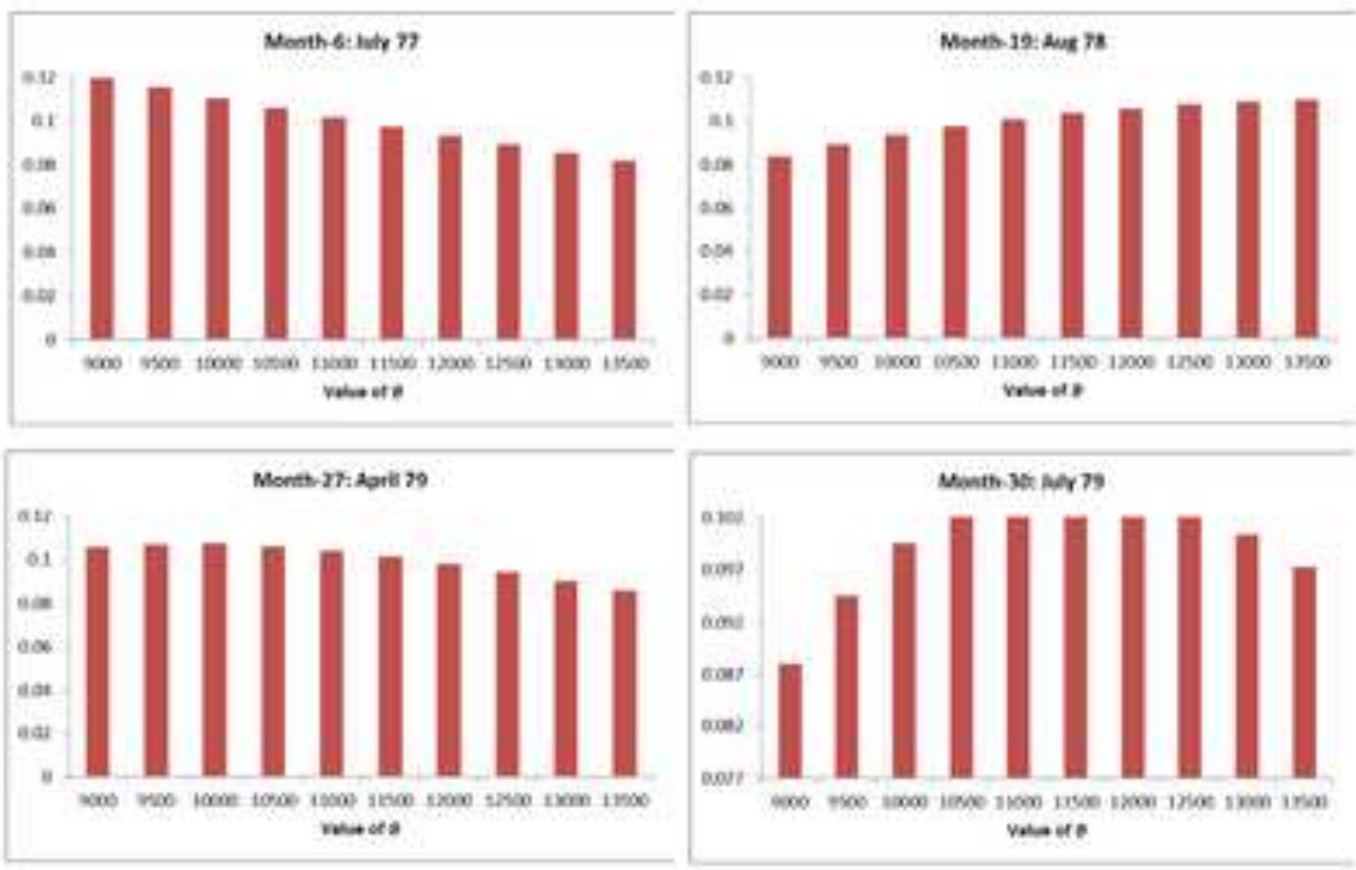

(b) Sigma $=2.0$ 

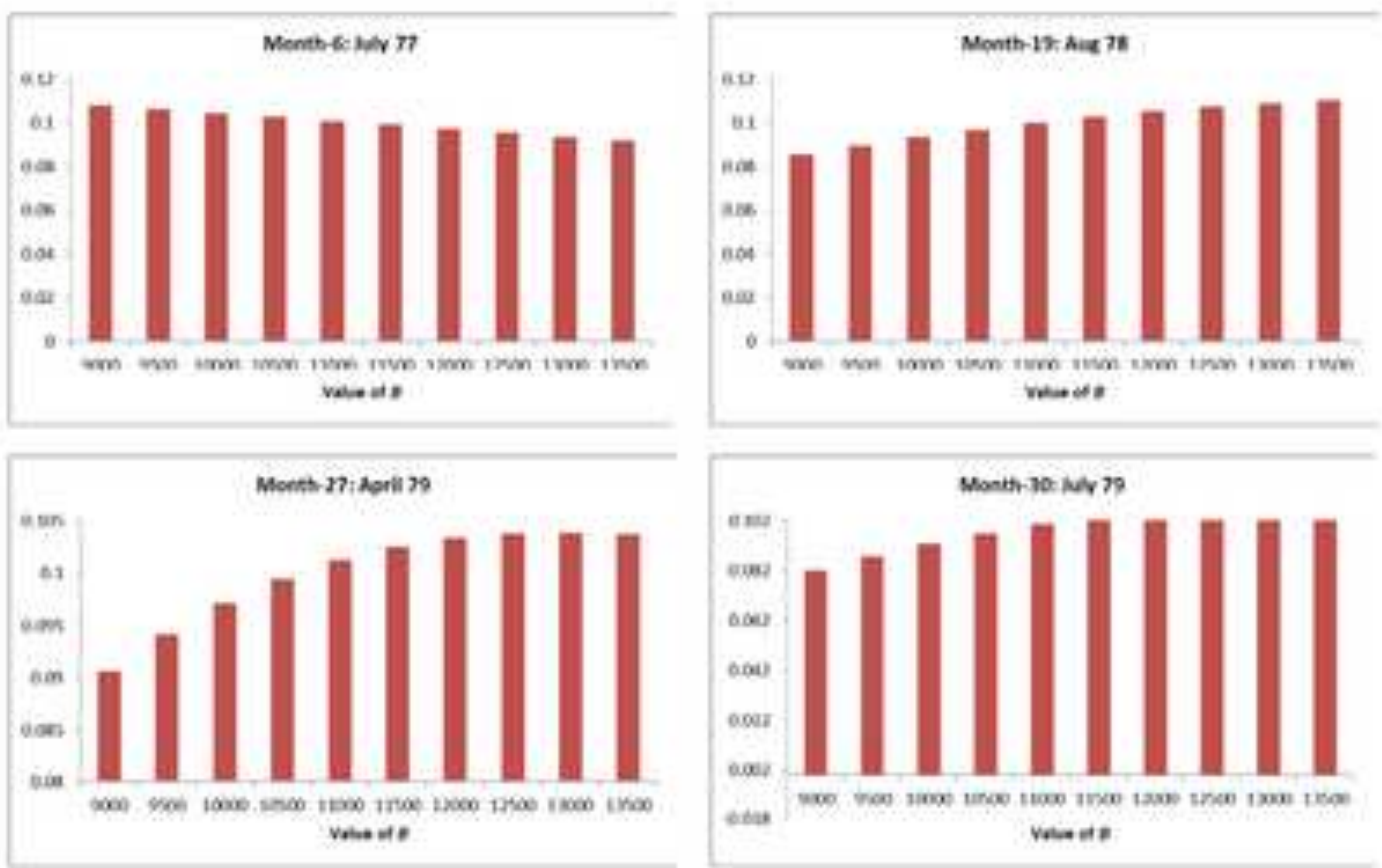

(c) Sigma $=3.0$ 

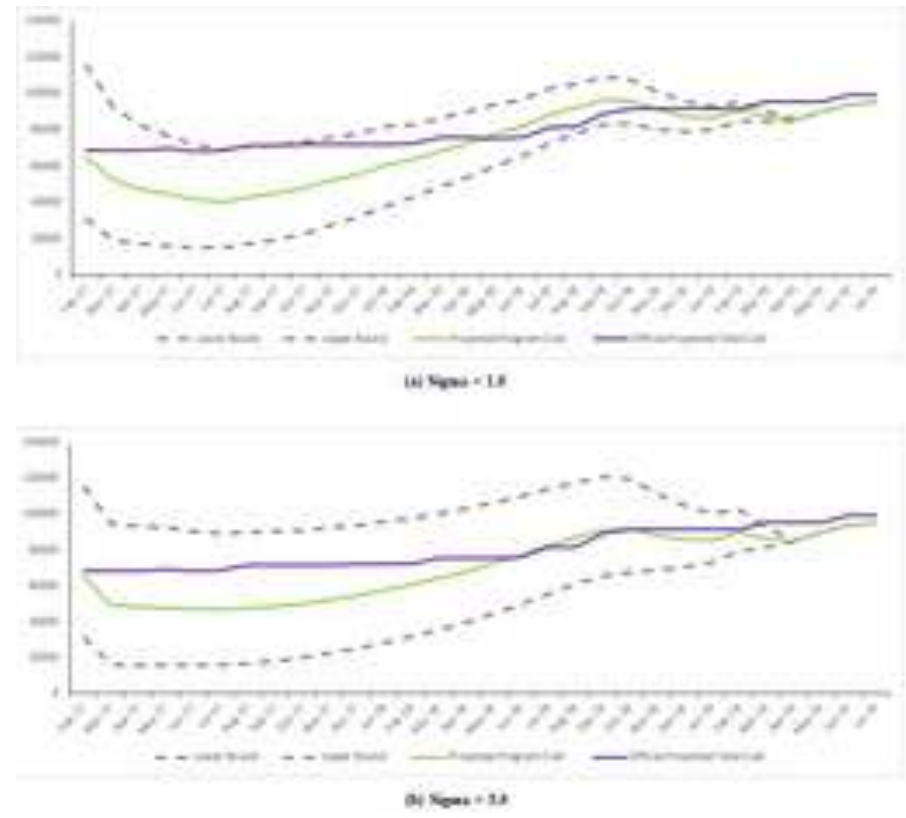